\title{
Raman Induced Visible Stable Platicons and Breather Platicons in Microresonator
}

\author{
Shunyu Yao ${ }^{1}$, Chengying Bao ${ }^{2}$ and Changxi Yang ${ }^{*}$ \\ ${ }^{1}$ State Key Laboratory of Precision Measurement Technology and Instruments, Department of Precision Instruments, Tsinghua University, \\ Beijing 100084, China \\ ${ }^{2}$ T. J. Watson Laboratory of Applied Physics, California Institute of Technology, Pasadena, California 91125, USA \\ Author e-mail address: cxyang@tsinghua.edu.cn
}

\begin{abstract}
We numerically demonstrate that stable platicons and coherent visible Kerr combs can be generated via Raman assisted four wave mixing in a AlN microresonator. Raman induced breather platicon dynamics is also observed in our simulations.(C) 2019 The Author(s)

OCIS codes: (190.4390) Nonlinear optics, integrated optics; (190.5530) Pulse propagation and temporal solitons; (140.3948) Microcavity devices
\end{abstract}

\section{Introduction}

Kerr frequency combs generated in continuous wave driven microresonators via cascade four-wave-mixing (FWM) have attracted widespread interest over the past decade [1]. However, most of the reported Kerr frequency combs operate in the near-infrared region, despite the urgent demand of coherent visible frequency combs. The challenge of generating coherent visible Kerr frequency combs lies in the strong normal material group velocity dispersion (GVD) in the visible wavelength, which makes it difficult to initiate comb generation via modulation instability (MI). Stable dark solitons or platicons (i.e., flat-topped pulse [2]) generation with normal dispersion has been reported, with the aid of mode-interaction [2, 3]. However, it requires an appropriate strength of mode-interaction, which is naturally hard to control [4]. On the other hand, in crystalline microresonators such as magnesium fluoride, silicon, diamond and aluminum nitride (AlN), the Raman gain is strong and narrow, and Kerr-Raman combs can be generated via Raman assisted four wave mixing (RFWM) process in those microresonators [5]. It is notable that RFWM process occur in both normal and anomalous dispersion, providing an opportunity to generate coherent visible combs. However, can stable platicon and coherent visible Kerr combs be generated via RFWM remains an open question.

Here we numerically show that RFWM is a feasible way to obtain coherent visible comb with normal dispersion. We obtain stable platicons and coherent visible combs with $780 \mathrm{~nm}$ pump in an AlN microresonator with the generalized Lugiato-Lefever equation (GLLE). Furthermore, Raman induced breather platicon is predicted for the first time, to our knowledge. The breather exhibits power exchange between the comb lines near the pump and the lines around the Raman gain peak. Our results provide new insights into the interaction between the SRS and the Kerr effect in microresonator and suggest that RFWM constitutes a feasible way to achieve coherent visible platicon combs with normal GVD.

\section{Simulation results}

The microresonator used here is composed of AlN-on-sapphire film. The dispersion of a $1400 \mathrm{~nm} \times 800 \mathrm{~nm}$ (width $\times$ height) AlN waveguide is calculated with a finited-element mode (FEM) solver. The waveguide shows a normal dispersion $\left(\beta_{2}=51.5 \mathrm{ps}^{2} / \mathrm{km}\right)$ at the pump wavelength $(780 \mathrm{~nm})$. The comb generation dynamics is simulated using GLLE; and the Raman effect is calculated in the frequency domain with a Lorentzian gain spectrum [6], whose peak is centered at $\Omega_{R} / 2 \pi=-18.3 \mathrm{THz}$ and FWHM is $\Gamma_{R} / 2 \pi=138 \mathrm{GHz}$ [5].
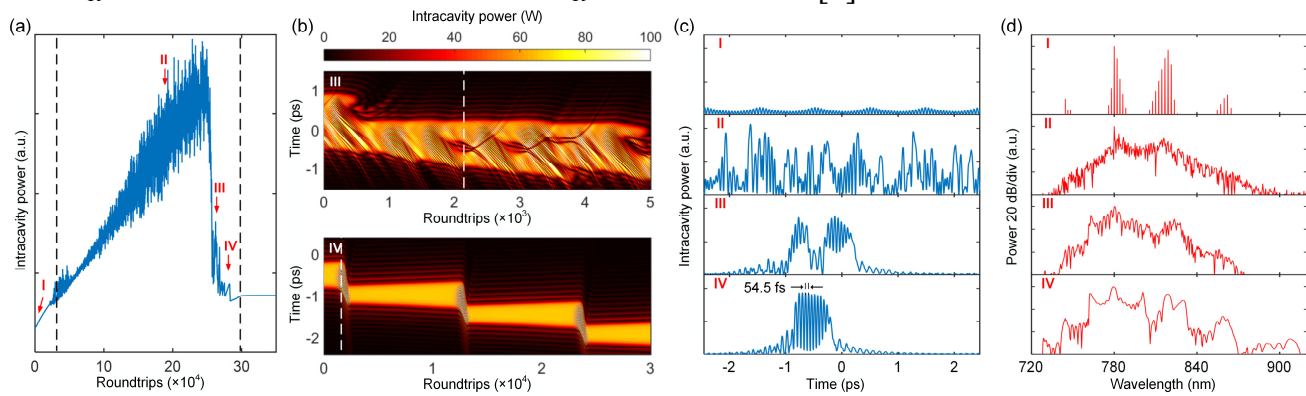

Fig. 1 Dynamics of Kerr-Raman comb generation in an AlN microresonator. The width of the waveguide is $1400 \mathrm{~nm}$ and the height is $800 \mathrm{~nm}$. (a) Evolution of the intracavity power when the pump is boosted to $660 \mathrm{~mW}$ and tuned into the resonance. (b) Evolution of the temporal intensity profile of position III and IV in (a). (c) Temporal waveform and (d) optical spectrum for different positions I-IV in (a). The exact position in the evolution is marked by dashed white line in (b). 
In the simulation, we start by a condition where the pump power is $123.8 \mathrm{~mW}$ and the detuning is -0.0072 . The Kerr-Raman comb stably exists in this state; the corresponding temporal waveform and optical spectrum are shown in part I of Figs. 1(c, d). We boost the pump to $660 \mathrm{~mW}$ at the 32600th round-trip (the first dashed black line in Fig. 1(a)) and then rapidly increase the detuning to 0.0636 at the 29910th round-trip (marked by the second dashed black line in Fig. 1(a)). The intracavity waveform becomes chaotic and a structured single FSR comb is obtained (see Part II of Figs. 1(c, d)). As the pump laser is tuned to about 0.057 , the intracavity power undergo a sharp decrease. During this power drop, part of the chaotic waveform survives and keeps circulating in the cavity. Fig. 1(b) III shows the evolution of this chaotic pulse when the detuning is kept as 0.0587 , corresponding to the marker III in Fig. 1(a). Part III of Figs. 1(c, d) shows the waveform and optical spectrum of the exact moment marked by white dashed line in Fig. 1(b) III; pulse-like waveform starts to form but with chaotic features.
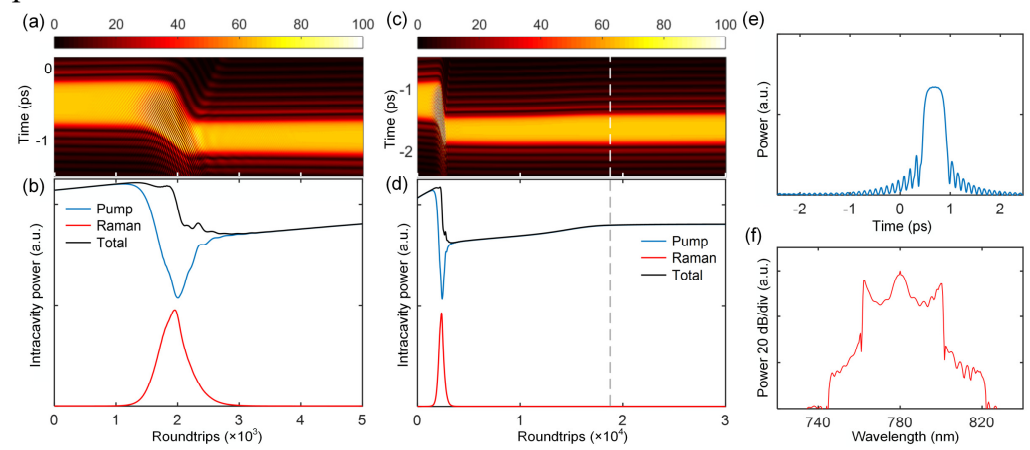

Fig. 2 (a) Temporal intensity evolution and (b) the corresponding power transfer between pump area and Raman area near the negative trajectory slope regime. (c) Temporal intensity evolution and (d) the corresponding power evolution during the transition from platicon breather to stable platicon. (e) Temporal waveform and (f) optical spectrum of the stable platicon.

When $\delta_{0}$ increases to 0.0629 , a pulsating flat-topped pulse emerges. Figure. $1(\mathrm{~b})$ IV shows the corresponding temporal waveform evolution. At first, the width of the pulse increases during evolution. Then the pulse trajectory shows a negative slope in the retarded fast time window, suggesting an increase group velocity. In this regime, the pulse waveform exhibits strong oscillations with a period of 54.5 fs (see Fig. 1(c) IV), which matches the Raman gain peak frequency 18.3 THz. Strong comb lines near the Raman gain peak on the spectrum rise simultaneously (see Fig. 1(d) IV). Then the negative slope of the pulse trajectory vanishes and the pulse becomes flat top without oscillations again. We zoom in the first 5000 round-trips of the temporal evolution in Fig. 2 (a) to gain more insight of the dynamics near the negative trajectory slope regime. The corresponding power evolution of the pump area $(760-800 \mathrm{~nm}$ of the spectrum), Raman area $(800-840 \mathrm{~nm})$ and the total intracavity during the transition is depicted in Fig. 2(b). The pump area power grows at first, providing more Raman gain for the Raman area. When the Raman gain exceeds the loss, the Raman area power grows rapidly at the expense of the pump area power. When the pump area power decreases further, the Raman gain decreases and the power returns to the pump area. The power exchange between the pump area and the Raman area is similar to Fermi-Pasta-Ulam recurrence induced by breather solitons in microresonators with anomalous dispersion [7]. Therefore, we classify the described phenomenon as Raman induced breather platicon, which is predicted for the first time, to our knowledge.

When we tune the detuning linearly from $\delta_{0}=0.0629$ to 0.0636 , the Kerr-Raman comb become stable and the comb manifest as a stable flat-topped pulse (platicon) in the time domain. Figure. 2(c) depicts the temporal intensity profile evolution of this transition. The corresponding evolution of the pump area power, the Raman area power and the total intracavity power are shown in Fig. 2(d). Dashed lines in Fig. 2(c) and Fig. 2(d) depict the moment where $\delta_{0}$ is tuned to 0.0636 . Figure. 2(e) shows the waveform of the stable platicon and its spectrum is shown in Fig. 2(f). The bandwidth of the comb is more than $80 \mathrm{~nm}$ with a $60 \mathrm{~dB}$ comb-line power change.

In summary, we obtain stable platicons and coherent visible combs with $780 \mathrm{~nm}$ pump in an AlN microresonator with normal dispersion. Generation dynamics of Raman induced breather platicon is predicted for the first time. Our results provide a new way to achieve coherent visible platicon combs with normal GVD.

\section{References}

[1] P. Del'Haye, A. Schliesser, O. Arcizet, T. Wilken, R. Holzwarth, and T. J. Kippenberg, Nature 450, 1214 (2007).

[2] V. Lobanov, G. Lihachev, T. Kippenberg, and M. Gorodetsky, Opt. Express 23, 7713-7721 (2015).

[3] X. Xue, Y. Xuan, Y. Liu, P.-H. Wang, S. Chen, J. Wang, D. E. Leaird, M. Qi, and A. M. Weiner, Nat. Photonics 9, 594 (2015).

[4] C. Bao, Y. Xuan, D. E. Leaird, S. Wabnitz, M. Qi, and A. M. Weiner, Optica 4, 1011-1015 (2017).

[5] X. Liu, C. Sun, B. Xiong, L. Wang, J. Wang, Y. Han, Z. Hao, H. Li, Y. Luo, and J. Yan, Optica 4, 893-896 (2017).

[6] Q. Lin, O. J. Painter, and G. P. Agrawal, Opt. Express 15, 16604-16644 (2007).

[7] C. Bao, J. A. Jaramillo-Villegas, Y. Xuan, D. E. Leaird, M. Qi, and A. M. Weiner, Phys. Rev. Lett. 117, 163901 (2016). 